\title{
1 A chimeric KaiA-like regulator extends the nonstandard KaiB3-KaiC3 2 clock system in bacteria
}

4 Christin Köbler ${ }^{a, 1}$, Nicolas M. Schmelling ${ }^{\mathrm{b}, 1}$, Alice Pawlowski ${ }^{\mathrm{b}, 1}$, Philipp Spät ${ }^{\mathrm{c}}$, Nina M. Scheurer ${ }^{\mathrm{a}}$,

5 Lutz Berwanger ${ }^{b}$, Boris Maček ${ }^{c}$, llka M. Axmann ${ }^{b}$, Annegret Wilde ${ }^{a, *}$

6 alnstitute of Biology III, Faculty of Biology, University of Freiburg, 79104 Freiburg, Germany;

7 bInstitute for Synthetic Microbiology, Biology Department, Heinrich Heine University Düsseldorf,

840225 Düsseldorf, Germany; 'Department of Quantitative Proteomics, Interfaculty Institute for

9 Cell Biology, Eberhard Karls University Tübingen, 72076 Tübingen, Germany

$10 \quad{ }^{1}$ C.K., N.M.S and A.P. contributed equally to this work.

11 *Prof. Dr. Annegret Wilde, Albert-Ludwigs-Universität Freiburg, Institut für Biologie III,

12 Schänzlestr. 1, 79104 Freiburg, Germany, Phone: +49 (0) 761-20397828

13 Email: annegret.wilde@biologie.uni-freiburg.de

14 Author Contributions: C.K., I.M.A. and A.W. designed the study. C.K., N.M.S., A.P., P.S., 15 N.M.Sche. and L.B. performed and analyzed the experiments. All authors interpreted and 16 discussed the data. C.K., N.M.S., A.P., P.S., B.M., I.M.A and A.W. wrote the paper.

17 Competing Interest Statement: The authors declare no competing interest.

18 Keywords: cyanobacteria, circadian clock, Synechocystis 6803, KaiA

19 This PDF file includes:

Main Text

Figures 1 to 4 


\section{Abstract}

The rotation of the Earth results in predictable environmental changes that pose challenges for organisms and force them to adapt. To address this daily rhythm, organisms from all kingdoms of life have evolved diverse timing mechanisms. In the cyanobacterium Synechococcus elongatus PCC 7942, the three proteins KaiA, KaiB, and KaiC constitute the central timing mechanism that drives circadian oscillations. In addition to the standard oscillator, named KaiAB1C1, Synechocystis sp. PCC 6803 harbors several, diverged clock homologs. The nonstandard KaiB3-KaiC3 system was suggested to impact the metabolic switch in response to darkness. Here, we demonstrate the direct interaction of KaiC3 with SII0485, which is a potential new chimeric KaiA homolog that we named KaiA3. The existence of a functional link between these proteins is further supported by the co-occurrence of genes encoding KaiA3 with the KaiB3-KaiC3-like gene products in 10 cyanobacterial and five other bacterial species. KaiA3 is annotated as a NarL-type response regulator due to its similarity to the response regulator receiver domains. However, its similarity to canonical NarL drastically decreases in the $\mathrm{C}$-terminal domain, which resembles the circadian clock protein KaiA. In line with this, we detected the stimulation of KaiC3 phosphorylation by KaiA3 in vitro. Furthermore, we showed that deletion of the kaiA3 gene led to growth defects during mixotrophic growth conditions and, like a kaiC3-deficient mutant, viability was impaired during chemoheterotrophic growth in complete darkness. In summary, we suggest KaiA3 as a novel, nonstandard KaiA homolog within the cyanobacterial phylum, extending the KaiB3-KaiC3 system in Cyanobacteria and other prokaryotes.

\section{Significance Statement}

Many organisms that are subjected to day and night cycles are able to predict daily changes using a circadian clock system. Cyanobacteria use a relatively simple timekeeper, a protein-based oscillator consisting of KaiA, KaiB and KaiC. Several cyanobacteria and other prokaryotes harbor additional sets of oscillator components that, however, lack the KaiA protein. Our study identified a divergent KaiA homolog that is functionally and genetically linked to potential KaiB3-KaiC3 timekeepers. The data show that the nonstandard KaiA homolog affects the phosphorylation of KaiC3 from the cyanobacterium Synechocystis, suggesting that a similar function exists in other prokaryotes.

\section{Main Text \\ Introduction}

Most organisms on Earth are subjected to predictable environmental changes, such as light and darkness or temperature variations during the day and night cycle. To adapt to these ever-changing conditions, eukaryotic and prokaryotic organisms have evolved endogenous biological clock systems.

To date, cyanobacteria are the only prokaryotes with a well-understood and robust circadian rhythm. The model organism for cyanobacterial circadian clocks, Synechococcus elongatus PCC 7942 (hereafter Synechococcus), encodes three genes, kaiA, kaiB, and kaiC, that constitute the core oscillator (1). The KaiC protein forms a homohexamer and has autokinase, autophosphatase, ATP synthase, and ATPase activities (1-4). Through binding to KaiC, KaiA stimulates the autokinase and ATPase activities of KaiC and thus the protein is phosphorylated (57). Upon phosphorylation of two neighboring residues (Ser431 and Thr432), KaiC undergoes structural rearrangements, exposing a binding site for KaiB (8-10). After binding, KaiB sequesters KaiA from KaiC, promoting KaiC's autophosphatase activity and the protein reverts back to its unphosphorylated state $(8,9,11)$. The interplay of KaiA and KaiB is crucial for the KaiC phosphorylation pattern, which in turn confers the clock phase and rhythmicity to the cell $(12,13)$. 
In Synechococcus, the KaiA protein functions as a homodimer and harbors two distinct domains connected by a linker sequence (14-16). The $\mathrm{N}$-terminal domain is similar to bacterial response regulators but lacks the aspartate residue crucial for phosphorylation; hence, it is designated as a pseudoreceiver domain (PsR domain) (14). This domain was shown to bind the oxidized form of quinones and is therefore able to directly sense the onset of darkness and forward signals to the C-terminal domain $(14,17)$. The C-terminus has a four-helix bundle secondary structure and is highly conserved within Cyanobacteria. The domain harbors the KaiA dimer interface and the KaiC binding site and is necessary to stimulate the autophosphorylation activity of $\operatorname{KaiC}(14,16)$. Mutations of kaiA, resulting in altered periodicity, were mapped throughout both domains, showing their importance for rhythmicity $(16,18)$. For a more detailed review on the KaiABC oscillator and its regulatory network, see Cohen and Golden (19), Swan et al. (20) and Snijder and Axmann (21). The circadian timing mechanism of Synechococcus is well understood; however, the composition of Kai systems can vary largely in other cyanobacteria and different clock sets, and their functions are less elucidated (22-25). Synechocystis sp. PCC 6803 (hereafter Synechocystis) harbors, in addition to the canonical kaiAB1C1 gene cluster, two further kaiB and kaiC homologs (26). The proteins KaiA, KaiB1, and KaiC1 seem to constitute the main timing mechanism, occupying the canonical SasA-RpaA output-signaling pathway $(27,28)$. Deletion of either kaiAB1C1 or rpaA led to decreased viability in light-dark cycles compared to that of the wild-type (WT) strain $(27,29)$. This phenotype was aggravated under photomixotrophic conditions and in complete darkness. In contrast, the function of the additional Kai homologs has rarely been investigated, and multiple copies of kaiA have not been identified thus far in any cyanobacterial strain (22).

For the Synechocystis KaiB3-KaiC3 timing system, Aoki and Onai suggested a function in the finetuning of the core oscillator KaiAB1C1 by modulating its amplitude and period (30). This idea was supported by Wiegard et al., who recently investigated the characteristics of the KaiC3 protein and proposed interplay between the KaiB3-KaiC3 system and the proteins of the standard clock system (31). Phenotypical analysis hinted at similar cellular functions of both timing systems. While deletion of kaiC3 had no effect on growth in light-dark cycles, the cells displayed growth defects under chemoheterotrophic conditions compared to that of the WT $(29,31)$. The impairment was less severe in comparison with a $\triangle$ kaiAB1C1-deficient strain, which completely lost the ability to grow in the dark. Furthermore, Wiegard et al. verified KaiA-independent autophosphorylation, autodephosphorylation, and ATPase activity of Synechocystis KaiC3; enzymatic activities suggested to be conserved in all cyanobacterial KaiC proteins $(22,31)$. However, compared to Synechococcus KaiC, KaiC3 ATPase activity was found to be diminished and lacked temperature compensation, an essential feature of true circadian oscillations $(2,31)$.

To date, the regulatory network of the KaiB3-KaiC3 system has remained enigmatic, as it does not interact with KaiA and does not utilize the SasA-RpaA output pathway, suggesting alternative, not yet identified, components for KaiB3-KaiC3-based signal transduction (27). In a large-scale proteinprotein interaction screen, a potential interaction partner of KaiC3 was identified (32). This protein, SII0485, was categorized as a NarL-type response regulator and could be a potential element in the KaiB3-KaiC3 signaling pathway (33).

Within this study, we characterized Sll0485 bioinformatically, highlighting a resemblance of the protein's N-terminal domain with the receiver domain of NarL-type response regulators, yet the C-terminal domain shared similarities to KaiA homologs. Furthermore, we showed computationally strong co-occurrences of the KaiB3-KaiC3 system with SII0485 in the genomic context of Cyanobacteria and other bacteria. We verified that the physical interaction of KaiC3 with SII0485 increased the phosphorylation activity of KaiC3. Thus, we propose that SII0485 is a novel KaiA-like homolog linked to the KaiB3-KaiC3 system. Deletion of s/l0485 led to impaired viability during nonphotoautotrophic growth, in line with previous studies on the KaiB3-KaiC3 system (31). Hence, we introduced the name KaiA3 for SII0485 and will use it from here onwards. Furthermore, to 
facilitate the distinction of the KaiA homologs, we will use the name KaiA1 for the Synechocystis KaiA core clock homolog SIr0756.

\section{Results}

KaiA3 is a chimeric protein harboring a NarL-type response regulator domain at the $N$-terminus and a conserved KaiA-like motif at the $C$-terminus

The canonical clock genes kaiABC and kaiA1B1C1 form a cluster in Synechococcus and Synechocystis, respectively. In contrast, the kaiB3 and kaiC3 genes of Synechocystis are localized in different regions of the chromosome (Fig. 1B, Fig. S1). Here, the kaiB3 gene forms a transcriptional unit with the open reading frame sll0485, encoding a hypothetical protein that we named KaiA3. This hypothetical protein is annotated as a NarL-type response regulator (33) yet displays a chimeric structure.

Using reciprocal BLAST analyses, we detected KaiA3 orthologs in 15 cyanobacterial species, mainly belonging to the order Chroococcales ((34); Data S1), and in five bacterial genera outside of Cyanobacteria, namely, Roseiflexus, Chloroflexus, Chloroherpeton, Rhodospirillum, and Bradyrhizobium.

Analysis of the KaiA3 amino acid sequences from all cyanobacterial species encoding this protein revealed that the $\mathrm{N}$-terminus is conserved and indeed shows high homology to NarL-type response regulators (Fig. S2). The canonical NarL protein consists of an $\mathrm{N}$-terminal receiver domain, a linker and a C-terminal DNA-binding domain with a helix-turn-helix motif $(33,35)$. For KaiA3, the similarities to the NarL protein decreased in the C-terminus (Fig. S2), and concurrently, conservation between KaiA3 and the KaiA protein family increased (Fig. 1A). The conserved residues in the $\mathrm{C}$-terminus correspond to structurally important features in the Synechococcus KaiA protein, such as a-helical secondary structures, the KaiA dimer interface or residues critical for the KaiA-KaiC interaction (Fig. 1A, 14, 15). Additionally, the lack of conservation in the N-terminus compared to that seen in known KaiA orthologs is consistent with the results of Dvornyk and Mei, which propose that different $\mathrm{N}$-terminal domains exist for KaiA homologs for functional diversification (36).

Multiple sequence alignments of the C-termini of the canonical KaiA and KaiA3 were performed to construct a gene tree to further elucidate the relationship of KaiA3 and KaiA orthologs. The resulting gene tree (Fig. 1A) distinctly separated KaiA3 from canonical KaiA orthologs. To further investigate the evolutionary relationship of KaiA3, multiple sequence alignments of the C-terminus of orthologs of KaiA3, KaiA, and SIr1783 (Rre1) as a reference for NarL orthologs in Cyanobacteria (37) were used to construct a phylogenetic tree (Fig. S3). Here, KaiA3 orthologs form a distinct clade at the basis of the KaiA orthologs when compared to both orthologous groups of SIr1783 (Rre1)/NarL ( $E$. coli, UniProtKB - POAF28) and KaiA simultaneously (Fig. S3). In summary, these findings strengthen the idea that the C-terminus of KaiA3 has a similar function to KaiA.

We further constructed three-dimensional models of KaiA3 to gain a better understanding of its potential functions. To date, no structure is available for KaiA3, and it was impossible to generate a reliable three-dimensional model covering the full-length KaiA3 sequence due to the enigmatic structure of the linker region for which no significant similarities could be detected. Thus, we modeled the $\mathrm{N}$-terminus (residues 1-140) and the remaining part of the sequence separately (residues 141-299). For the $\mathrm{N}$-terminus, numerous hits for response regulator domains were obtained, confirming previous findings. The 3D-model structures of KaiA3 are highly similar and display the canonical fold of response regulator domains: a central five-stranded parallel $\beta$-sheet flanked on both faces by five amphipathic $\alpha$-helices and a phosphorylatable aspartate residue in the 33 -strand (Fig. S2, Fig. S4). This aspartate residue (D65), important for phosphorylation in NarL-type response regulators (Fig. S2, blue stars), is conserved in all but Pleurocapsa and Microcystis species. Thus, most KaiA3 homologs, including the Synechocystis protein, harbor a potential phosphorylation site. Furthermore, the structure superimposes well on the PsR domain of KaiA, even though the PsR domain lacks the aspartate residue that can be phosphorylated and the 
$\alpha 4-$ helix between the $\beta 4$ - and $\beta 5$-strands (Fig. S4). The amino acid sequence between the $\beta 4$ - and $\beta 5$-strands shows the least conservation between KaiA and KaiA3, yet the level of sequence conservation in this region is generally low for KaiA and its homologs (16). In contrast to the $\mathrm{N}$-terminal response regulator domain, the $\mathrm{C}$-terminal domain of KaiA3 revealed a unique fold, which has only been detected in KaiA thus far (38) and the N-terminal domain of the phosphoserine phosphatase RsbU from Bacillus subtilis (39), namely, a unique four a-helix bundle constituting the KaiA-like motif (Fig. S5). In conclusion, we propose that KaiA3 consists of two protein modules with i) the $\mathrm{N}$-terminal domain, resembling an NarL-type response regulator receiver domain, including its phosphorylation site, and ii) the C-terminal domain displaying features of a KaiA-like motif. This is particularly intriguing since putative kaiA orthologs outside of Cyanobacteria have not been identified until recently (36).

Conserved synteny and co-occurrence of KaiA3 and the KaiB3-KaiC3 system among prokaryotes As in Synechocystis, we found the kaiA3 gene upstream of kaiB3 in all analyzed cyanobacterial genomes. Furthermore, the kaiA3B3 cluster is usually extended by kaiC3 with only two exceptions (Synechocystis and Microcystis aeruginosa NIES-843), resembling the structure of the canonical kaiABC gene cluster (Fig. 1B). Interestingly, kaiA3B3C3 synteny is also found in other prokaryotic genomes that harbor orthologs of kaiA3, except for Chloroflexus aggregans DMS 9485 (Fig. 1B). Furthermore, we detected strong significant co-occurrences between KaiA3 and KaiB3 $(p<0.0001)$, as well as KaiA3 and KaiC3 ( $p<0.0001$; Fig. 1C). The co-occurrence of KaiB3 and KaiC3 has already been shown previously (22). Thus, KaiA3 forms a distinct set of proteins with KaiB3 and KaiC3 that shows no further significant co-occurrence with other clock components (Fig. 1C, (22). Altogether, both data sets suggest a functional relationship between KaiA3 and the KaiB3-KaiC3 system.

\section{KaiC3 interacts with the hypothetical protein KaiA3}

Using yeast two-hybrid experiments, we verified an interaction between the clock proteins KaiC3 and KaiA3 (Fig. 2A), consistent with the previous protein-protein interaction analysis by Sato et al. (32). Although KaiA3 clearly interacts with KaiC3, an interaction with KaiB3, the second element of the KaiB3-KaiC3 clock system, was not detected (Fig. S6). To further characterize the interaction of the proteins in vitro, we expressed them recombinantly in $E$. coli and analyzed the complex formation using native PAGE (Fig. 2B). KaiA3-His6 (monomer: $35 \mathrm{kDa}$ ) migrated as one single band of approximately $100 \mathrm{kDa}$ size, confirming the capability of KaiA3 to form homodimers. Recombinant KaiB3 (monomer: $12 \mathrm{kDa}$ ) was recently shown to form dimers and tetramers of 13 and $41 \mathrm{kDa}$ size, respectively, after size exclusion chromatography (31). KaiB3 displayed three distinct bands in native gels. The two lower bands most likely represent the dimeric and tetrameric forms, while the uppermost band $(\sim 67 \mathrm{kDa})$ is probably an impurity in the protein preparation. Recombinant KaiC3 was produced with an N-terminal Strep-tag (31). Strep-KaiC3 (monomer: $58 \mathrm{kDa}$ ) migrated as one band at $\sim 400 \mathrm{kDa}$, in line with the size of the hexamer (348 kDa). Interestingly, this band vanishes completely when KaiC3 is incubated with KaiA3 (Fig. 2B). Instead, protein accumulates in the well of the gel, indicating precipitation of the KaiA3/KaiC3 complex. On the other hand, the formation of a large protein complex was observed when all three clock components, KaiA3, KaiB3 and KaiC3, were incubated together for $16 \mathrm{~h}$ at $30^{\circ} \mathrm{C}$. The size matches a complex consisting of one KaiC3 hexamer, six KaiA3 dimers and six KaiB3 monomers (840 kDa). The presence of KaiA3 in the complex was validated by Western blotting using an anti-His antibody (Fig. 2C). As expected, no such complex formed when KaiA3 was replaced by Synechococcus KaiA (Fig. 2B). The interaction of KaiA3 and KaiC3 could, in addition, be validated using immunoprecipitation-coupled liquid chromatography-mass spectrometry (LC-MS) analysis of FLAG-tagged KaiC3 (Fig. S8). Supplementary experiments did not reveal any interaction between KaiA3 and KaiC1 or KaiC2 (Fig. S6, Fig. S9), implying specificity for KaiC3 exists.

KaiA3 stimulates the autokinase activity of KaiC3

Previous studies showed that KaiC3 has autokinase activity and that this activity is independent of KaiA $(28,31)$. Since our studies revealed an interaction of KaiC3 with KaiA3, we were interested in 
probing the influence of KaiA3 on the phosphorylation of KaiC3. For this purpose, the abovedescribed recombinant Kai proteins were used. KaiC3 was incubated at $30^{\circ} \mathrm{C}$ in the presence or absence of other Kai proteins, and its phosphorylation state was analyzed by SDS-PAGE or LC-MS/MS (Fig. 3). KaiC3 purified from E. coli was predominantly phosphorylated (Fig. 3A). During the course of incubation, KaiC3 autodephosphorylates as is typical for KaiC proteins (39). KaiC3-dephosphorylation is enhanced by KaiB3. In contrast, the addition of KaiA3 keeps KaiC3 in the phosphorylated state, similar to the Synechococcus KaiC/KaiA core oscillator (6). The KaiA3mediated stimulation of KaiC3 phosphorylation was even more obvious when dephosphorylated KaiC3 (NP-KaiC3) was used in the assay (Fig. 3B). Analysis of KaiC3 phosphorylation by LC-MS/MS identified the neighboring residues Ser423 and Thr424 as phosphorylation sites, which are conserved across KaiC homologs. However, only singly phosphorylated peptides could be identified, with either Ser423 or Thr424 being phosphorylated. Overall, phosphorylation site localization probabilities were higher for Ser423 (84\%) compared to Thr424 (57\%), defining Ser423 as the primary phosphorylation site at present assay conditions (Fig. S10). In line with SDSPAGE analyses, consecutive phosphorylation occupancy analysis of the corresponding peptides revealed low phosphorylation levels throughout most incubation conditions, except for enhanced phosphorylation when KaiC3 was coincubated with KaiA3 for $16 \mathrm{~h}$ (Fig. 3C, Data S3). Interestingly, an additional, low abundant phosphorylation event could be identified on Thr321, which however remained at low occupancy $(<1 \%)$ at all incubation conditions.

As expected, Synechococcus KaiA did not influence the phosphorylation of KaiC3 (Fig. S11). Based on these analyses, we conclude that KaiA3 likely has a KaiA-like function in promoting the phosphorylation of KaiC3.

Deletion of kaiA3 impacts growth and viability during mixotrophic and chemoheterotrophic growth As KaiA3 shows significant co-occurrence with $\mathrm{KaiB} 3$ and $\mathrm{KaiC} 3$, as well as in vitro interaction with KaiC3, an interconnected function of the three proteins is more than likely. Deletion of kaiC3 led to growth impairments in complete darkness on glucose, but not in light-dark cycles (31); thus, the kaiA3 knockout mutant ( $\triangle$ kaiA3) was analyzed under these growth conditions. Cells were grown in liquid culture under constant light, plated on agar in different dilutions and grown photoautotrophically and photomixotrophically in continuous light and in 12-h light/12-h dark cycles (Fig. 4A) or chemoheterotrophically (Fig. 4B). As the strains grow very slowly in chemoheterotrophic conditions, cells were spotted at higher concentrations under these conditions. There were no differences in viability of the mutant strains in comparison to the WT under photoautotrophic conditions in continuous light and in light/dark cycles. Under photomixotrophic conditions, the $\triangle$ kaiA3 strain showed less viability, and viability was partly restored by re-insertion of kaiA. A mutant strain, lacking all three alternative kai genes $(\triangle$ kaiA3B3C3) grew similar to the WT. However, in light/dark cycles this strain seemed to have some advantage in comparison to the WT (Fig. 4A). Spot assays under chemoheterotrophic conditions gave a clearer picture: the mutant strain lacking kaiA3 and the triple knockout showed a similar phenotype. They were unable to grow in complete darkness and in the complementation strain this ability was fully restored (Fig. 4B). These results coincide with the previously detected impairments displayed by the $\Delta$ kaiC3 strain during chemoheterotrophic growth (31), strengthening the idea that the alternative kai3 genes form a regulatory complex with a common function.

\section{Discussion}

Despite increasing knowledge on circadian rhythms in Cyanobacteria and other prokaryotes, information on the function, composition, and network of (cyano)bacterial nonstandard clock systems remains scarce. By identifying KaiA3 and verifying its interaction with the KaiB3-KaiC3 complex, we could add another component to nonstandard clock systems. 
286 Recently, new putative KaiA orthologs have been bioinformatically identified in prokaryotes outside of Cyanobacteria (36). Even though multiple copies of the core clock proteins KaiB and KaiC have been found to be encoded in cyanobacterial genomes, canonical KaiA was only found as a single copy. In phylogenetic reconstructions, KaiA3 forms a distinct clade at the basis of the KaiA clade. Together with the results of the structural analyses, interesting questions are raised about the evolution of the KaiABC-based circadian clock system. It remains elusive whether KaiA3 together with KaiB3 and KaiC3 represents an ancestral system for the well-studied Synechococcus circadian clock, and further in-depth analyses are needed to elucidate the evolution of this new putative timing system. Furthermore, together with KaiB3 and KaiC3, which have already been found in other bacteria, including Rhodospirillum rubrum, where rhythmic processes have been described (41), KaiA3 homologs could represent an overlooked component of Kai-based systems outside of Cyanobacteria.

\section{The two-domain architecture of KaiA3 and complex formation}

KaiA3 displays a modular structure with an $\mathrm{N}$-terminal response regulator domain connected via a linker sequence to a C-terminal KaiA-like domain. The high structural similarity of the N-terminal domain to numerous response regulator domains from various organisms indicates that the core structure and activity are maintained, while adaptivity and variation from this provide specificity for acting in distinct pathways (30). Within KaiA3, the aspartate residue crucial for phosphorylation is conserved; thus, theoretically, the protein could receive an input signal from a cognate histidine kinase, which has not been identified thus far.

The nearly unique structure of the KaiA C-terminus is, in addition to KaiA, solely found in the $\mathrm{N}$-terminal domain of the phosphatase RsbU of Bacillus subtilis (39). RsbU acts as a positive regulator of the alternative sigma factor $B$, which is involved in the general stress response (42). Interestingly, the $\mathrm{N}$-terminal domain of RsbU forms dimers similar to KaiA, and the proposed binding site for its corresponding activator RsbT is in an equivalent location to the KaiC-binding site on KaiA (39). These findings may reflect how protein domains change during evolution while their original functions are conserved. Compared to KaiA, in KaiA3, only five out of the 13 residues for KaiC interaction are conserved (Fig. 1). However, KaiC3 also shows, in comparison to the KaiC1 homolog, less conservation of the residues important for the interaction with KaiA (28), perhaps due to coevolution.

The KaiA3 structures obtained from models based on NarL and KaiA superimpose neatly on the crystal structure of the KaiA dimer (Fig. S5, D), yet the composition of the linker subsequent to the a5-helix (E141-D178) remains cryptic. In KaiA, the canonical linker, represented by residues G165-K173, carries a $\beta$-sheet $\left(\beta_{6}\right)$ crucial for the KaiA-KaiB interaction $(43,44)$. Native PAGE analysis indicates that a complex forms when all three KaiA3, KaiB3 and KaiC3 clock components are present but there is no complex between KaiA3 and KaiB3 (Fig. 2B). However, Synechococcus CikA also lacks such a $\beta$-sheet but is able to form a complex with KaiB via its PsR domain (44). Our analyses clearly imply that KaiA3 binds KaiC3 (Fig. 2, Fig. S8), which induces KaiC3 phosphorylation (Fig. 3). Therefore, the proteins form a stable complex only together with KaiB3, otherwise aggregation occurs (Fig. 2B). Taken together, the data are consistent with a model in which KaiA3 is able to fulfill the functions of a KaiA homolog, such as dimerization, by binding to the KaiC homolog and enhancing autophosphorylation. Other mechanistic processes, such as sequestration to the $\mathrm{Cl}$ ring by binding to a KaiB homolog, remain to be investigated but are clearly possible.

\section{The function of KaiA3 within the cell}

The physiological function of KaiA3 seems to be related to the different metabolic modes of Synechocystis. This cyanobacterial strain is able to use glucose as an energy and carbon source in the light as well as in the dark. It should be noted that the Synechocystis WT strain used in this study was able to grow in complete darkness when supplemented with glucose. This is different from an earlier study that showed that Synechocystis needs a 5 min blue-light pulse at least once a day to be able to grow heterotrophically in the dark (45). The authors described this kind of behavior as light-activated heterotrophic growth. There are no studies that explain why cells need 
this 5 min light pulse, but it is also clear that our laboratory strain grows fully chemoheterotrophically (29). Mutants deficient in kaiA3 lose the ability to grow chemoheterotrophically on glucose, which is an aggravated effect, compared to kaiC3-deficient mutants, which merely showed reduced growth rates during heterotrophy (31). Similarly, in Synechococcus, the disruption of kaiA led to one of the most severe mutations regarding activity loss and was traced back to an unbalanced output signaling of the circadian clock (46). Related phenotypes, such as impaired viability during light-dark cycles, including the complete loss of chemoheterotrophic growth on glucose, were observed for the Synechocystis mutants $\triangle$ kaiA1B1C1, $\Delta$ sasA, and $\triangle r p a A(29,47)$. For $\triangle s a s A$, it was shown that the mutant strain was able to accumulate glycogen but was incapable of utilizing the storage compound to grow heterotrophically, probably due to the inability to catabolize glucose (47). A recent metabolomics study suggested that the growth inhibition of the $\triangle$ kaiA1B1C1 and $\triangle r p a A$ mutants in a light-dark cycle might be at least partly related to a defect in the inhibition of the RuBisCo enzyme in the dark and increased photorespiration leading to the accumulation of the potentially toxic product 2-phosphoglycolate (48). This previous study also revealed an enhanced growth defect of the $\triangle k a i A 1 B 1 C 1$ and $\triangle r p a A$ mutants under photomixotrophic conditions in light/dark cycles, similar to the $\triangle$ kaiA3 strain in the current study. This further supports the idea that one of the functions of the KaiA3B3C3 system is to fine-tune the core clock system KaiA1B1C1. Based on the conclusions from our studies, we propose that KaiA3 is a novel, nonstandard KaiA homolog and element of the KaiC3-based signaling pathway. KaiA3 most likely adopts the function of a KaiA homolog. The $\mathrm{N}$-terminal half of KaiA3 might still have a response regulator function, yet the exact mechanism remains unclear. Among other actions, KaiA3 needs to be placed within the regulatory and metabolic network.

\section{Materials and Methods}

Reciprocal BLAST of SII0485 (KaiA3) and SIr1783 (Rre1)

Reciprocal BLAST was performed as described in Schmelling et al., 2017 (22). For comparison with existing data about other circadian clock proteins, the 2017 database was used. The protein sequences of SII0485 (KaiA3) and SIr1783 (Rre1), as a reference for NarL response regulators (37) from Synechocystis were used as query sequences for this reciprocal BLAST.

\section{Synteny analyses using SyntTax}

The conservation of gene order was analyzed with the web tool 'SyntTax' (53; https://archaea.i2bc.paris-saclay.fr/synttax). If not mentioned otherwise default settings (Best match, $10 \%$ norm. BLAST) were applied. Chromosomes were selected manually according to the results of Schmelling et al. (2017).

\section{Multiple sequence alignments with Mafft and Jalview}

Sequence alignments, visualization, and analysis were performed with 'Jalview' (50). The sequences were aligned with Mafft, and if not mentioned otherwise, default settings (L-INS-i, pairwise alignment computation method -- localpair using Smith-Waterman algorithm, gap opening penalty: 1.53 , gap opening penalty at local pairwise alignment: -2.00 , group-to-group gap extension penalty: 0.123 , matrix: BLOSUM62) were applied (51). For the analyses of the C-terminus, the alignments were trimmed to position 168 in the KaiA reference sequence of Synechococcus. After trimming, the alignment was recalculated with Mafft using the abovementioned default parameters.

\section{$2 D$ and $3 D$ structure predictions}

The alignments generated in Jalview were then used with 'Ali2D' for secondary structure prediction (56; https://toolkit.tuebingen.mpg.de). The identity cutoff to invoke a new PSIPRED run was set to $30 \%$. Three-dimensional protein structures were modeled with either Phyre2 or SWISS-MODEL (58, 59; http://www.sbg.bio.ic.ac.uk/phyre2/html/page.cgi?id=index; 
https://swissmodel.expasy.org/). The resulting structures were then analyzed and illustrated using

\begin{abstract}
Phylogenetic reconstruction of protein trees
Phylogenetic reconstruction of the protein trees of SII0485 (KaiA3), SIr1783 (Rre1)/NarL (E. coli, UniProtKB - P0AF28), and KaiA was achieved with MEGA X $(56,57)$ using the above constructed alignments. For all alignments, a neighbor-joining tree and a maximum likelihood tree were constructed and compared. For the construction of the neighbor-joining trees, 1000 bootstrap iterations with a p-distance substitution model and a gamma distribution with three gamma parameters were used. For the construction of the maximum likelihood trees, an initial tree was constructed using the maximum parsimony algorithm. Further trees were constructed using 1000 bootstrap iterations with an LG-G substitution model, a gamma distribution with three gamma parameters and nearest-neighbor-interchange (NNI) as the heuristic method.
\end{abstract}

Yeast two-hybrid assay

AH109 yeast cells (Clontech) were used for the yeast two-hybrid experiments. Transformation of yeast cells was performed according to the manufacturer's guidelines using the Frozen-EZ Yeast Transformation kit (Zymo Research). The construction of plasmids used for transformation is described in the Supplementary Information. Successfully transformed cells were selected on complete supplement mixture (CSM) lacking leucine and a tryptophan (-Leu -Trp) dropout medium (MP Biochemicals) at $30^{\circ} \mathrm{C}$ for 3 - 4 days. Cells containing bait and prey plasmids were streaked on CSM lacking leucine, tryptophan and histidine (-Leu -Trp -His) dropout medium (MP Biochemicals) with the addition of $12.5 \mathrm{mM}$ 3-amino-1,2,4-triazole (3-AT, Roth) and incubated for 6 days at $30^{\circ} \mathrm{C}$ to screen for interaction.

\title{
Expression and purification of recombinant Kai proteins
}

Synechocystis KaiB3 and Synechococcus KaiA (plasmids kindly provided by T. Kondo, Nagoya University, Japan) were produced as GST-fusion proteins in E. coli BL21(DE3) as described (30; https://www.protocols.io/view/expression-and-purification-of-gst-tagged-kai-prot-48ggztw). Briefly, proteins were purified with affinity chromatography using glutathione-agarose 4B (Macherey and Nagel), and the N-terminal GST-tag was removed by PreScission Protease (Cytiva) prior to elution of the untagged proteins from the glutathione resin. Synechocystis KaiC3 was produced with an N-terminal Strep-tag (Strep-KaiC3) in E. coli Rosetta gami B (DE3) cells and purified via affinity chromatography using Strep-tactin XT superflow (IBA-Lifesciences)

https://www.protocols.io/view/heterologous-expression-and-affinity-purification-meac3ae). Synechocystis ORF sll0485, encoding KaiA3, was inserted into vector pET22b to create a C-terminal His6-fusion. KaiA3-His6 was expressed in E. coli Tuner(DE3) cells and purified with immobilized metal affinity chromatography (IMAC) using PureProteome ${ }^{\mathrm{TM}}$ Nickel Magnetic Beads (Millipore). For a detailed protocol see at protocols.io (dx.doi.org/10.17504/protocols.io.bu5bny2n). Recombinant proteins were stored at $-80^{\circ} \mathrm{C}$ in buffer containing $20 \mathrm{mM}$ Tris, $\mathrm{pH} 8.0,150 \mathrm{mM} \mathrm{NaCl}$, $0.5 \mathrm{mM}$ EDTA, $5 \mathrm{mM} \mathrm{MgCl}_{2}$, and $1 \mathrm{mM}$ ATP.

KaiC3 phosphorylation assay

Recombinant Strep-KaiC3 purified from E. coli mainly exists in its phosphorylated form (KaiC3-P). Fully dephosphorylated Strep-KaiC3 (KaiC3-NP) was generated by incubating the protein for $18 \mathrm{~h}$ at $30^{\circ} \mathrm{C}$ in assay buffer $(20 \mathrm{mM}$ Tris, $\mathrm{pH} 8.0,150 \mathrm{mM} \mathrm{NaCl}, 0.5 \mathrm{mM}$ EDTA, $5 \mathrm{mM} \mathrm{MgCl} 2$, and 1 mM ATP). The autokinase activity of KaiC3-NP was investigated by incubating $4 \mu \mathrm{g}$ KaiC3 for 16 $\mathrm{h}$ at $30{ }^{\circ} \mathrm{C}$ in $20 \mu \mathrm{l}$ assay buffer in the presence or absence of $2 \mu \mathrm{g}$ KaiA3-His6, KaiB3 and Synechococcus KaiA. Ten-microliter aliquots were taken before and after incubation at $30^{\circ} \mathrm{C}$, and the reaction was stopped with SDS sample buffer. Samples were applied to a LowC SDS gel (10\% $\mathrm{T}, \quad 0.67 \% \quad$ C) $(63 ;$ https://www.protocols.io/view/lowc-sds-page-gysbxwe) and stained with Coomassie Blue R.

For analysis of KaiC3 phosphorylation occupancy levels in in vitro coincubation by LC-MS/MS, proteins were digested in solution by chymotrypsin (Fig. 3C) or trypsin (Fig. S10), and measured 
on a Q Exactive HF mass spectrometer (ThermoFisher Scientific) as described before (59). Raw data was processed as described in supplementary methods with the following exceptions: MaxQuant version 1.6.8.0 was used and phosphorylation of Ser, Thr and Tyr was defined as a variable modification. Phosphorylation occupancy levels for each condition were calculated based on intensity sums of modified/(modified + unmodified) peptides containing phosphorylation sites Ser423 or Thr424 (Data S3)

\section{Native protein PAGE and immunodetection of KaiA3}

Kai proteins (10 $\mu \mathrm{l}$ samples containing $2 \mu \mathrm{g}$ dephosphorylated Strep-KaiC3, $1 \mu \mathrm{g}$ KaiA3-His6, $1 \mu \mathrm{g}$ KaiB3, $1 \mu \mathrm{g}$ Synechococcus KaiA) were incubated for $16 \mathrm{~h}$ at $30^{\circ} \mathrm{C}$ in phosphorylation assay buffer, followed by separation of the native proteins in $4-16 \%$ native PAGE at $4{ }^{\circ} \mathrm{C}$ using a clear native buffer system (Serva). Protein bands were visualized with Coomassie staining (ROTIBlue Quick, Carl Roth) or immunodetected with a monoclonal anti-His antibody conjugated to HRP (MA121315-HRP, Thermo Fisher, 1:2000 diluted). A detailed protocol is found at protocols.io (dx.doi.org/10.17504/protocols.io.bu67nzhn).

\section{Strains and growth conditions}

Wild type Synechocystis (PCC-M, resequenced, (60)), the deletion strains $\triangle r p a A(27), \Delta$ kaiC3 (31), $\triangle$ kaiA3 and $\triangle$ kaiA3B3C3 (SI, Fig. S1) and the complementation strain $\triangle$ kaiA3/kaiA3 (SI, Fig. S1) were cultured photoautotrophically in BG11 medium (61) supplemented with $10 \mathrm{mM}$ TES buffer (pH 8) under constant illumination with $75 \mu \mathrm{mol}$ photons $\mathrm{m}^{-2} \mathrm{~s}^{-1}$ of white light (Philips TLD Super $80 / 840)$ at $30^{\circ} \mathrm{C}$. Cells were grown either in Erlenmeyer flasks with constant shaking (140 rpm) or on plates ( $0.75 \%$ Bacto-Agar, Difco) supplemented with $0.3 \%$ thiosulfate. For photomixotrophic experiments, $0.2 \%$ glucose was added to the plates. For chemoheterotrophic growth experiments in complete darkness, Synechocystis cells were spotted in different dilutions on BG11 agar plates containing $0.2 \%$ glucose and incubated either mixotrophically for 3 days with continuous illumination or chemoheterotrophically in the dark for 26 days.

\section{Data availability}

The mass spectrometry proteomics data have been deposited to the ProteomeXchange Consortium (http://proteomecentral.proteomexchange.org) via the PRIDE partner repository (62) with the dataset identifier PXD027256.

Information for reviewers only (must be deleted in final manuscript): Reviewer account details: Username: reviewer_pxd027256@ebi.ac.uk; Password: eeeG1OAV

\section{Acknowledgments}

We thank Research Unit FOR2816 'SCyCode' (The Autotrophy-Heterotrophy Switch in Cyanobacteria: Coherent Decision-Making at Multiple Regulatory Layers) funded by the German Research Foundation for fruitful discussions and financial support. This work was financially supported by grants (WI2014/5-3; 10-1; AX 84/1-3 and MA 4918/4-1) from the German Research Foundation to A.W., I.M.A. and B.M. respectively. We thank Pauline Morys, Annika Klopp, and Werner Bigott for technical assistance.

\section{References}

1. M. Nakajima, et al., Reconstitution of circadian oscillation of cyanobacterial KaiC phosphorylation in vitro. Science. 308, 414-415 (2005).

2. K. Terauchi, et al., ATPase activity of KaiC determines the basic timing for circadian clock 
501 of cyanobacteria. Proc. Natl. Acad. Sci. U. S. A. 104, 16377-16381 (2007).

502

503

504

505

506

507

508

509

510

511

512

513

514

515

516

517

518

519

520

521

522

523

524

525

526

527

528

529

530

531

532

533

534

535

536

537

538

539

540

541

542

543

544

545

546

547

548

549

550

551

552

553

554

3. M. Egli, et al., Dephosphorylation of the Core Clock Protein KaiC in the Cyanobacterial KaiABC Circadian Oscillator Proceeds via an ATP Synthase Mechanism. Biochemistry 51, 1547-1558 (2012).

4. R. Pattanayek, et al., Visualizing a Circadian Clock Protein: Crystal Structure of KaiC and Functional Insights. Mol. Cell 15, 375-388 (2004).

5. Y. I. Kim, G. Dong, C. W. Carruthers, S. S. Golden, A. LiWang, The day/night switch in KaiC, a central oscillator component of the circadian clock of cyanobacteria. Proc. Natl. Acad. Sci. U. S. A. 105, 12825-12830 (2008).

6. H. Iwasaki, T. Nishiwaki, Y. Kitayama, M. Nakajima, T. Kondo, KaiA-stimulated KaiC phosphorylation in circadian timing loops in cyanobacteria. Proc. Natl. Acad. Sci. U. S. A. 99, 15788-93 (2002).

7. R. Pattanayek, M. Egli, Protein-Protein Interactions in the Cyanobacterial Circadian Clock: Structure of KaiA Dimer in Complex with C-Terminal KaiC Peptides at $2.8 \AA$ Resolution. Biochemistry 54, 4575-4578 (2015).

8. Y.-G. Chang, R. Tseng, N.-W. Kuo, A. LiWang, Rhythmic ring-ring stacking drives the circadian oscillator clockwise. Proc. Natl. Acad. Sci. U. S. A. 109, 16847-51 (2012).

9. Y.-G. Chang, N.-W. Kuo, R. Tseng, A. LiWang, Flexibility of the C-terminal, or Cll, ring of KaiC governs the rhythm of the circadian clock of cyanobacteria. Proc. Natl. Acad. Sci. U. S. A. 108, 14431-6 (2011).

10. T. Nishiwaki, et al., Role of KaiC phosphorylation in the circadian clock system of Synechococcus elongatus PCC 7942. Proc. Natl. Acad. Sci. U. S. A. 101, 13927-13932 (2004).

11. Y. Kitayama, H. Iwasaki, T. Nishiwaki, T. Kondo, KaiB functions as an attenuator of KaiC phosphorylation in the cyanobacterial circadian clock system. EMBO J. 22, 2127-2134 (2003).

12. R. Tseng, et al., Cooperative KaiA-KaiB-KaiC Interactions Affect KaiB/SasA Competition in the Circadian Clock of Cyanobacteria. J. Mol. Biol. 426, 389-402 (2014).

13. M. J. Rust, J. S. Markson, W. S. Lane, D. S. Fisher, E. K. O'Shea, Ordered phosphorylation governs oscillation of a three-protein circadian clock. Science 318, 809-12 (2007).

14. S. B. Williams, I. Vakonakis, S. S. Golden, A. C. LiWang, Structure and function from the circadian clock protein KaiA of Synechococcus elongatus: a potential clock input mechanism. Proc. Natl. Acad. Sci. U. S. A. 99, 15357-62 (2002).

15. I. Vakonakis, et al., NMR structure of the KaiC-interacting C-terminal domain of KaiA, a circadian clock protein: implications for KaiA-KaiC interaction. Proc. Natl. Acad. Sci. U. S. A. 101, 1479-84 (2004).

16. S. Ye, I. Vakonakis, T. R. loerger, A. C. LiWang, J. C. Sacchettini, Crystal structure of circadian clock protein KaiA from Synechococcus elongatus. J. Biol. Chem. 279, 20511-8 (2004).

17. Y. I. Kim, D. J. Vinyard, G. M. Ananyev, G. C. Dismukes, S. S. Golden, Oxidized quinones signal onset of darkness directly to the cyanobacterial circadian oscillator. Proc. Natl. Acad. Sci. U. S. A. 109, 17765-17769 (2012).

18. H. Nishimura, et al., Mutations in KaiA, a clock protein, extend the period of circadian rhythm in the cyanobacterium Synechococcus elongatus PCC 7942. Microbiology 148, 2903-2909 (2002).

19. S. E. Cohen, S. S. Golden, Circadian Rhythms in Cyanobacteria. Microbiol. Mol. Biol. Rev. 79, 373-385 (2015).

20. J. A. Swan, S. S. Golden, A. LiWang, C. L. Partch, Structure, function, and mechanism of the core circadian clock in cyanobacteria. J. Biol. Chem. 293, 5026-5034 (2018).

21. J. Snijder, I. M. Axmann, "The Kai-Protein Clock-Keeping Track of Cyanobacteria's Daily Life" in Subcellular Biochemistry, (Springer, 2019), pp. 359-391.

22. N. M. Schmelling, et al., Minimal tool set for a prokaryotic circadian clock. BMC Evol. Biol. 17, 169 (2017).

23. V. Dvornyk, O. Vinogradova, E. Nevo, Origin and evolution of circadian clock genes in 
prokaryotes. Proc Natl Acad Sci U S A 100, 2495-2500 (2003).

24. I. M. Axmann, S. Hertel, A. Wiegard, A. K. Dörrich, A. Wilde, Diversity of KaiC-based timing systems in marine Cyanobacteria. Mar. Genomics 14, 3-16 (2014).

25. V. Dvornyk, "The circadian clock gear in cyanobacteria: Assembled by evolution" in Bacterial Circadian Programs, (Springer-Verlag Berlin Heidelberg, 2009), pp. 241-258.

26. Y. Kanesaki, et al., Identification of Substrain-Specific Mutations by Massively Parallel Whole-Genome Resequencing of Synechocystis sp. PCC 6803. DNA Res. 19, 67-79 (2012).

27. C. Köbler, S. J. Schultz, D. Kopp, K. Voigt, A. Wilde, The role of the Synechocystis sp. PCC 6803 homolog of the circadian clock output regulator RpaA in day-night transitions. Mol. Microbiol. 110, 847-861 (2018).

28. A. Wiegard, et al., Biochemical analysis of three putative KaiC clock proteins from Synechocystis sp. PCC 6803 suggests their functional divergence. Microbiology 159, 948958 (2013).

29. A. K. Dörrich, J. Mitschke, O. Siadat, A. Wilde, Deletion of the Synechocystis sp. PCC 6803 kaiAB1C1 gene cluster causes impaired cell growth under light-dark conditions. Microbiology 160, 2538-2550 (2014).

30. S. Aoki, K. Onai, "Circadian clocks of Synechocystis sp. strain PCC 6803, Thermosynechococcus elongatus, Prochlorococcus spp., Trichodesmium spp. and other species" in Bacterial Circadian Programs, (Springer-Verlag Berlin Heidelberg, 2009), pp. 259-282.

31. A. Wiegard, et al., Synechocystis KaiC3 displays temperature- And KaiB-dependent ATPase activity and is important for growth in darkness. J. Bacteriol. 202, 1-36 (2020).

32. S. S. Sato, et al., A large-scale protein protein interaction analysis in Synechocystis sp. PCC 6803. DNA Res. 14, 207-16 (2007).

33. I. Baikalov, et al., Structure of the Escherichia coli response regulator NarL. Biochemistry 35, 11053-11061 (1996).

34. J. Komarek, J. Kaštovský, J. Mares, J. Johansen, Taxonomic classification of cyanoprokaryotes (cyanobacterial genera) 2014, using a polyphasic approach. Preslia Praha- 86, 295-335 (2014).

35. M. Y. Galperin, Structural classification of bacterial response regulators: Diversity of output domains and domain combinations. J. Bacteriol. 188, 4169-4182 (2006).

36. V. Dvornyk, Q. Mei, Evolution of kaiA, a key circadian gene of cyanobacteria. Sci. Rep. 11, 9995 (2021).

37. M. K. Ashby, J. Houmard, Cyanobacterial Two-Component Proteins: Structure,Diversity, Distribution, and Evolution. Microbiol. Mol. Biol. Rev. 70, 472-509 (2006).

38. I. Vakonakis, A. C. LiWang, Structure of the C-terminal domain of the clock protein KaiA in complex with a KaiC-derived peptide: implications for KaiC regulation. Proc. Natl. Acad. Sci. U. S. A. 101, 10925-30 (2004).

39. O. Delumeau, et al., Functional and structural characterization of RsbU, a stress signaling protein phosphatase 2C. J. Biol. Chem. 279, 40927-40937 (2004).

40. T. Nishiwaki, T. Kondo, Circadian autodephosphorylation of cyanobacterial clock protein KaiC occurs via formation of ATP as intermediate. J. Biol. Chem. 287, 18030-18035 (2012).

41. E. Van Praag, R. Degli Agosti, R. Bachofen, Rhythmic Activity of Uptake Hydrogenase in the Prokaryote Rhodospirillum rubrum. J. Biol. Rhythms 15, 218-224 (2000).

42. X. Yang, C. M. Kang, M. S. Brody, C. W. Price, Opposing pairs of serine protein kinases and phosphatases transmit signals of environmental stress to activate a bacterial transcription factor. Genes Dev. 10, 2265-2275 (1996).

43. J. Snijder, et al., Structures of the cyanobacterial circadian oscillator frozen in a fully assembled state. Science. 355, 1181-1184 (2017).

44. R. Tseng, et al., Structural basis of the day-night transition in a bacterial circadian clock. Science. 355, 1174-1180 (2017).

45. S. L. Anderson, L. McIntosh, Light-activated heterotrophic growth of the cyanobacterium Synechocystis sp. strain PCC 6803: A blue-light-requiring process. J. Bacteriol. 173, 2761- 
2767 (1991).

46. D. G. Welkie, et al., Genome-wide fitness assessment during diurnal growth reveals an expanded role of the cyanobacterial circadian clock protein KaiA. Proc. Natl. Acad. Sci. U. S. A. 115, E7174-E7183 (2018).

47. A. K. Singh, L. A. Sherman, Pleiotropic effect of a histidine kinase on carbohydrate metabolism in Synechocystis sp. strain PCC 6803 and its requirement for heterotrophic growth. J. Bacteriol. 187, 2368-76 (2005).

48. N. M. Scheurer, et al., Homologs of Circadian Clock Proteins Impact the Metabolic Switch Between Light and Dark Growth in the Cyanobacterium Synechocystis sp. PCC 6803. Front. Plant Sci. 12, 675227 (2021).

49. J. Oberto, SyntTax: A web server linking synteny to prokaryotic taxonomy. BMC Bioinformatics 14 (2013).

50. A. M. Waterhouse, J. B. Procter, D. M. A. Martin, M. Clamp, G. J. Barton, Jalview Version 2-A multiple sequence alignment editor and analysis workbench. Bioinformatics 25, 11891191 (2009).

51. K. Katoh, D. M. Standley, MAFFT multiple sequence alignment software version 7: Improvements in performance and usability. Mol. Biol. Evol. 30, 772-780 (2013).

52. L. Zimmermann, et al., A Completely Reimplemented MPI Bioinformatics Toolkit with a New HHpred Server at its Core. J. Mol. Biol. 430, 227-2243 (2018).

53. A. Waterhouse, et al., SWISS-MODEL: homology modelling of protein structures and complexes. Nucleic Acids Res. 46 (2018).

54. L. A. Kelley, S. Mezulis, C. M. Yates, M. N. Wass, M. J. E. Sternberg, The Phyre2 web portal for protein modeling, prediction and analysis. Nat. Protoc. 10, 845-858 (2015).

55. E. F. Pettersen, et al., UCSF Chimera - A visualization system for exploratory research and analysis. J. Comput. Chem. 25, 1605-1612 (2004).

56. S. Kumar, G. Stecher, M. Li, C. Knyaz, K. Tamura, MEGA X: Molecular evolutionary genetics analysis across computing platforms. Mol. Biol. Evol. 35, 1547-1549 (2018).

57. G. Stecher, K. Tamura, S. Kumar, Molecular Evolutionary Genetics Analysis (MEGA) for macOS. Mol. Biol. Evol. 37, 1237-1239 (2020).

58. T. Nishiwaki, et al., A sequential program of dual phosphorylation of KaiC as a basis for circadian rhythm in cyanobacteria. EMBO J. 26, 4029-4037 (2007).

59. P. Spät, T. Barske, B. Maček, M. Hagemann, Alterations in the $\mathrm{CO}_{2}$ availability induce alterations in the phosphoproteome of the cyanobacterium Synechocystis sp. PCC 6803. New Phytol. 231, 1123-1137 (2021).

60. D. Trautmann, B. Voß, A. Wilde, S. Al-Babili, W. R. Hess, Microevolution in cyanobacteria: Re-sequencing a motile substrain of Synechocystis sp. PCC 6803. DNA Res. 19, 435-448 (2012).

61. R. Rippka, J. Deruelles, M. Herdman, J. B. Waterbury, R. Y. Stanier, Generic Assignments, Strain Histories and Properties of Pure Cultures of Cyanobacteria. Microbiology 111, 1-61 (1979).

62. Y. Perez-Riverol, et al., The PRIDE database and related tools and resources in 2019: improving support for quantification data. Nucleic Acids Res. 47, D442-D450 (2019). 
bioRxiv preprint doi: https://doi.org/10.1101/2021.07.20.453058; this version posted July 21, 2021. The copyright holder for this preprint (which was not certified by peer review) is the author/funder. All rights reserved. No reuse allowed without permission.

653

654
Figures and Tables

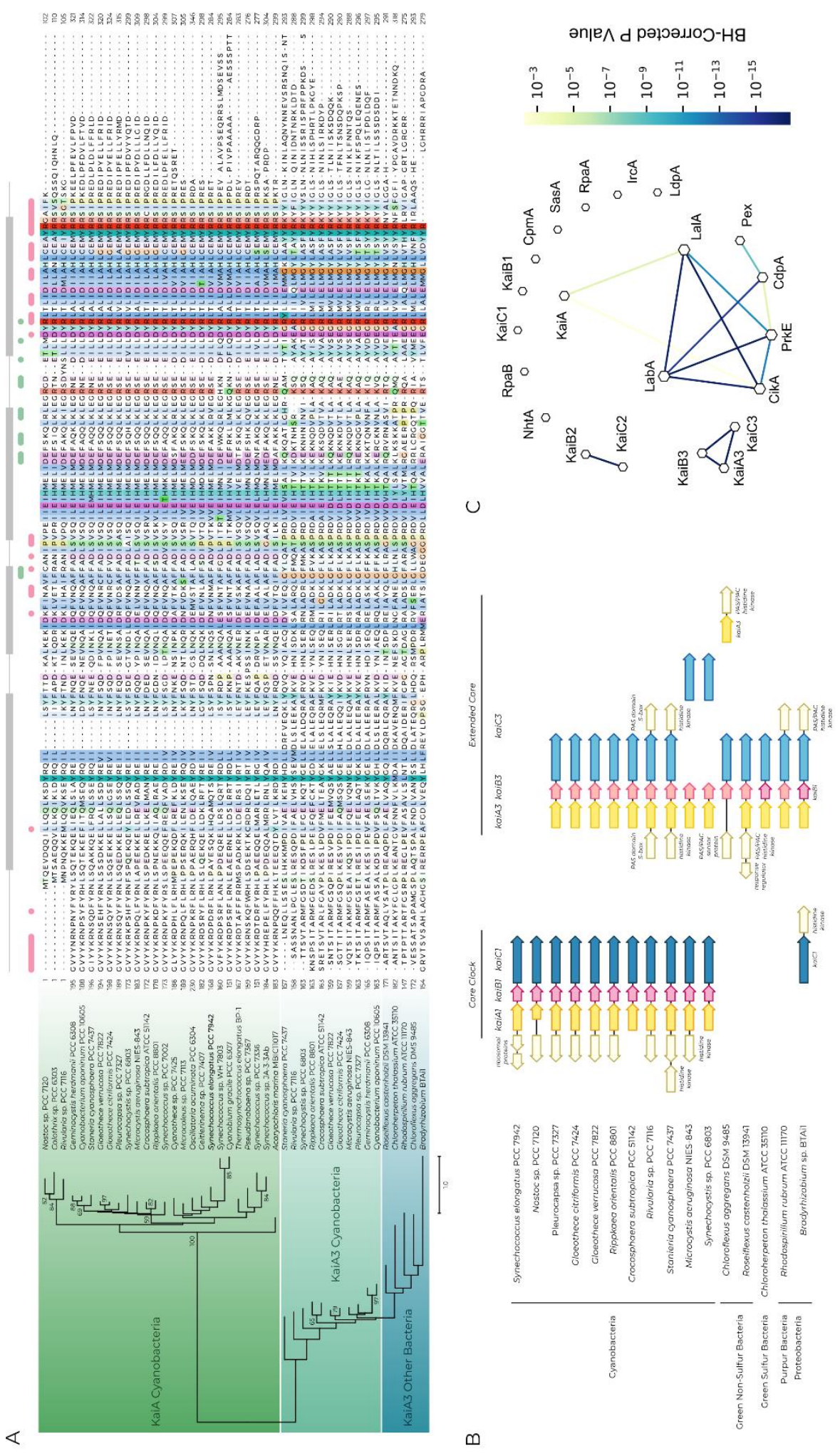


bioRxiv preprint doi: https://doi.org/10.1101/2021.07.20.453058; this version posted July 21, 2021. The copyright holder for this preprint (which was not certified by peer review) is the author/funder. All rights reserved. No reuse allowed without permission.

656 Fig. 1. Bioinformatic analyses of KaiA3. (A) Multiple sequence alignment and maximum likelihood657 inferred phylogenetic reconstruction of KaiA3 and selected KaiA orthologs. The sequences were 658 aligned with Mafft (L-INS-i default parameters, Jalview), trimmed to position 168 of the C-terminus 659 of Synechococcus KaiA and are represented in the Clustalx color code with conservation visibility 660 set to $25 \%$. Marks above the alignment refer to Synechococcus KaiA as a reference. Light green 661 bars and dots indicate residues critical for KaiC interaction, light pink bars and dots represent 662 residues important for dimerization, and light gray blocks outline residues forming a-helices as 663 secondary structures. Aligned sequences were used to infer a maximum likelihood protein tree. 664 The scale bar indicates 1 substitution per position. Bootstrap values $(n=1000)$ are displayed at 665 branches. Bootstrap values less than 50 are not shown. (B) Synteny analysis of KaiABC compared 666 to KaiA3, KaiB3, and KaiC3 for selected bacterial species. Analysis was performed with the online 667 tool SyntTax, a prokaryotic synteny and taxonomy explorer (https://archaea.i2bc.paris668 saclay.fr/synttax/; 2020-06-08). Default settings were used for analysis (best match, 10\% norm. 669 Blast). (C) Co-occurrence of KaiA3 using pairwise Fisher's exact tests with circadian clock proteins in Cyanobacteria. Network of significant co-occurring circadian clock factors from Schmelling et al. (22) including KaiA3 in Cyanobacteria. The line color corresponds to the level of significance resulting from the pairwise Fisher's exact test. Missing links are those that had a p-value higher than 0.01 . Node size is proportional to the degree of that node. 
676

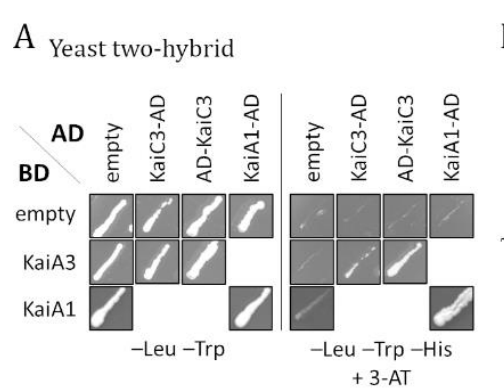

Fig. 2. Analysis of KaiA3 protein interactions. (A) Yeast two-hybrid interaction analysis of KaiA3 with the KaiC3 protein. As a positive control, KaiA1 dimer interaction was used. Yeast two-hybrid reporter strains carrying the respective bait, and prey plasmids were selected by plating on complete supplement medium (CSM) lacking leucine and tryptophan (-Leu -Trp). AD, GAL4 activation domain; BD, GAL4 DNA-binding domain; empty, bait and prey plasmids without protein sequence (only $A D / B D$ domain). The physical interaction between bait and prey fusion proteins was determined by growth on complete medium lacking leucine, tryptophan and histidine (-Leu -Trp -His) and the addition of $12.5 \mathrm{mM}$ 3-amino-1,2,4-triazole (3-AT). BD was fused to the $\mathrm{N}$-terminus of KaiA3. For clear presentation, spots were assembled from several assays (original scans are shown in Fig. S7). (B), (C) Interaction analysis of recombinant Kai proteins in native polyacrylamide gels. Proteins were incubated for $16 \mathrm{~h}$ at $30{ }^{\circ} \mathrm{C}$ and subsequently subjected to 4-16\% clear native PAGE. Gels were either stained with Coomassie Blue (B) or blotted and immunodecorated with a monoclonal anti-His antibody for the detection of recombinant KaiA3-His6 (C). Recombinant Synechococcus KaiA was used for comparison. 


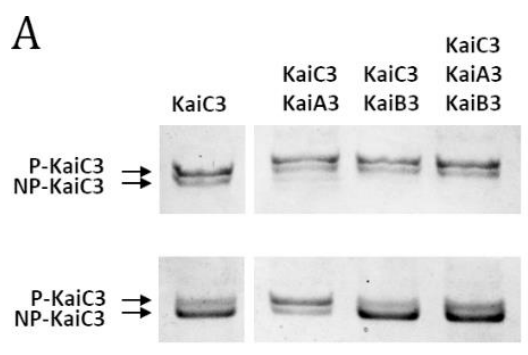

694

695

696

697

698

699

700

701

702

703

704

705

706

707

708 of nonmodified peptides only.
$\mathrm{B}$

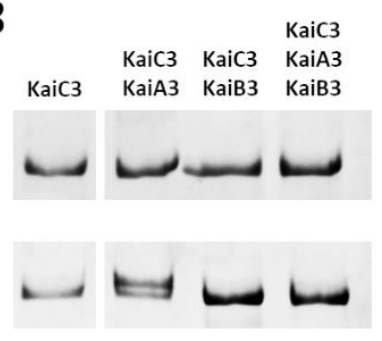

$16 \mathrm{~h}$

Fig. 3. KaiA3-stimulated phosphorylation of KaiC3. KaiC3 was incubated at $30^{\circ} \mathrm{C}$ with or without KaiA3 and KaiB3. Aliquots were taken at $0 \mathrm{~h}$ and $16 \mathrm{~h}$ followed by separation of phosphorylated (P) and nonphosphorylated (NP) KaiC3 on SDS-PAGE (10\% T, 0.67\% C). (A) Phosphorylated KaiC3 was used as the starting material for the assay. (B) Nonphosphorylated KaiC3 generated by an $18 \mathrm{~h}$ incubation at $30^{\circ} \mathrm{C}$ prior to the start point was used as the material for the assay. (C) Analysis of KaiC3 phosphorylation levels by LC-MS/MS. Samples were prepared as in (B) and digested in solution with chymotrypsin. Phosphorylation occupancy was calculated for each sample (in \%) based on intensity sums of modified/(modified + unmodified) peptides containing phosphorylation sites Ser423 or Thr424 (marked in bold; phosphopeptides exclusively singly phosphorylated). Sequences and start/end positions of detected peptides are indicated. *detection 
A
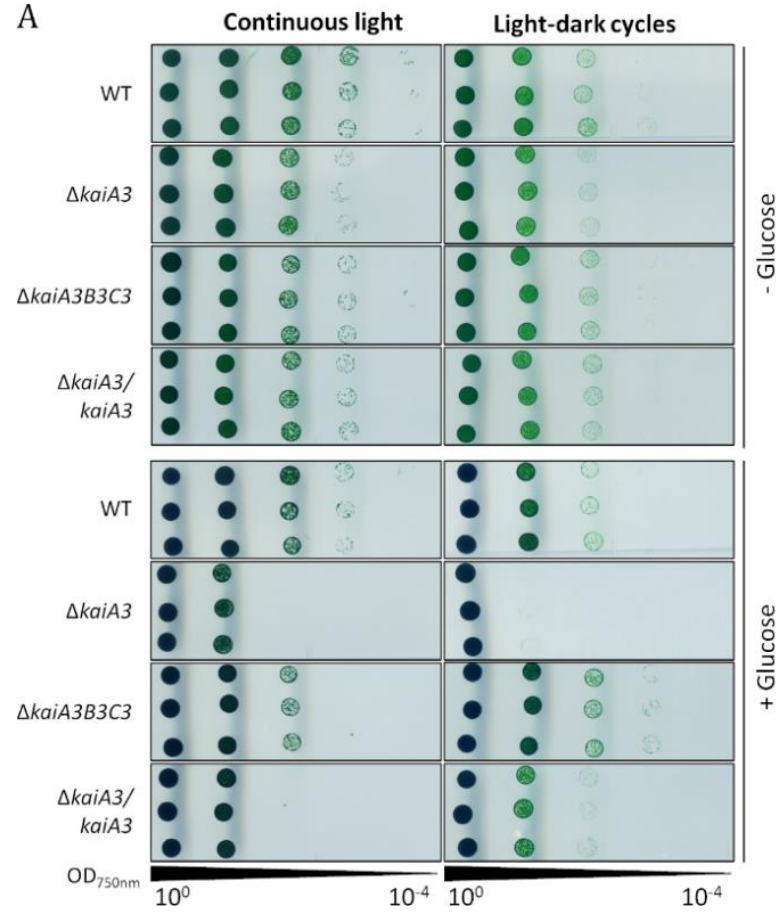

B

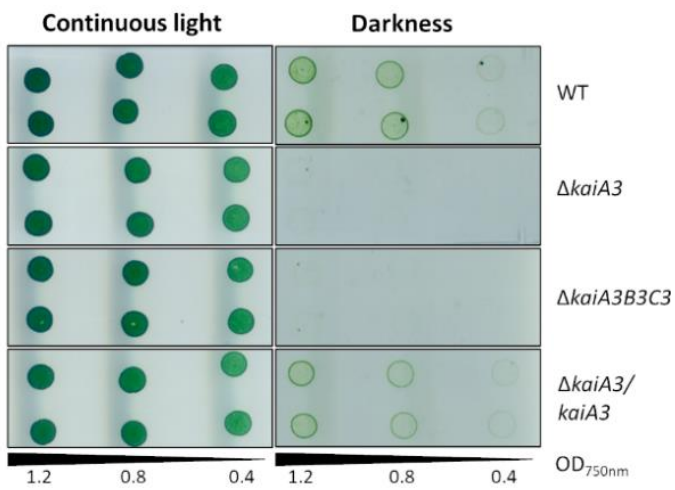

Fig. 4. Deletion of kaiA3 results in growth defects during mixotrophic and chemoheterotrophic growth. Proliferation of the WT, the kaiA3 and kaiA3B3C3 deletion mutant, and the $\triangle$ kaiA3/kaiA3 complementation strain was tested under phototrophic (continuous light, - glucose) photomixotrophic (continuous light, + glucose) and heterotrophic (darkness, + glucose) conditions. Strains were grown in liquid culture under constant light, different dilutions were spotted on agar plates and incubated in the indicated light conditions with light phase corresponding to $75 \mu \mathrm{mol}$ photons $\mathrm{m}^{-2} \mathrm{~s}^{-1}$ white light. A representative result of three independent experiments is shown. (A) Cultures were diluted to $O_{750 \mathrm{~nm}}$ value 0.4 and dilution series were spotted on agar plates with or without the addition of glucose. Plates were analyzed after 6 or 8 days of continuous light and $12 \mathrm{~h} / 12 \mathrm{~h}$ light-dark cycles, respectively. (B) Cultures were diluted to $\mathrm{OD}_{750 \mathrm{~nm}}$ values of $1.2,0.8$ and 0.4 and spotted on agar plates supplemented with $0.2 \%$ glucose. Plates were analyzed after 3 or 26 days of continuous light and darkness, respectively. 